\title{
METODOLOGIAS Y HERRAMIENTAS PARA LA SEGURIDAD DEL PACIENTE
}

\author{
METODOLOGIES AND TOOLS FOR \\ THE SAFETY OF THE PATIENT
}

Recibido noviembre 2016 Aceptado diciembre 2016

Correspondencia:

E.E.A.E.C. Sara Reyes Bautista Calle 7, No. 262, Colonia Agrícola Pantitlán C.P. 08100, delegación Iztacalco

Ciudad de México Teléfono: 04455 10456459, 57639593

Mail: s_reyes72@hotmail.com

E.E.A.E.C Luna García Mirna Nallely Emiliano Zapata No. 23, Colonia Luis Donaldo Colosio, C.P. 55010 Ecatepec de Morelos, Estado de México Teléfono: 0445554635018 Mail: nallemextligar@hotmail.com

\section{Autores:}

Benítez Ramos Stefany Itzel

Estudiante del Programa de Maestría en Enfermería de la Universidad Nacional Autónoma de México; Ciudad de México, México

Brito Carbajal Carlos Emmanuel

Estudiante del Programa de Maestría en Enfermería de la Universidad Nacional Autónoma de México; Ciudad de México, México

Domínguez Miguel Abigail

Estudiante del Programa de Maestría en Enfermería de la Universidad Nacional Autónoma de México; Ciudad de México, México

Luna García Mirna Nallely

Estudiante del Programa de Maestría en Enfermería de la Universidad Nacional Autónoma de México; Ciudad de México, México

Reyes Bautista Sara

Estudiante del Programa de Maestría en Enfermería de la Universidad Nacional Autónoma de México; Ciudad de México, México

\section{Espinal García María Del Pilar}

Estudiante del Programa de Maestría en Enfermería de la Universidad Nacional Autónoma de México; Ciudad de México, México

Zárate Grajales Rosa Amarilis

Profesor de carrera de tiempo completo del Programa de Maestría en Enfermería de la Escuela Nacional de Enfermería y Obstetricia de la Universidad Nacional Autónoma de México; Ciudad de México, México

Palabras clave: gestión de riesgos, enfermería, seguridad del paciente

Key words: risk management, patient safety, nursing 


\section{RESUMEN}

Trabajar en la calidad y seguridad de la atención, aumenta la satisfacción del paciente en los servicios de salud, reduce costos y salva vidas. La preocupación por la seguridad del paciente aunque no es tema nuevo, requiere seguir trabajando en la implementación de las metodologías y herramientas de la gestión de riesgos y documentar los resultados y las experiencias obtenidas. La poca evidencia científica documentada en México, propone una gran área de oportunidad para que los profesionales de enfermería contribuyan a través de la implementación de la gestión de riesgos, la investigación, y la documentación de los hallazgos, en el fortalecimiento de la práctica en enfermería y de esta manera al desarrollo de ésta como ciencia.

Palabras clave: gestión de riesgos, enfermería, seguridad del paciente.

\section{ABSTRACT}

Enhencing the safety and quality of the attention improves the patient's satisfaction towards health services, reduces costs and reduces mortality. The patients safety care, although not a new topic, needs to be kept adressing, methodology and tools implementation of risk management, as well as, to record data and achieved experiences. Very few scientific evidence gathered in Mexico provides a wide opportunity for nurse professionals to contribute through the implementation of risk management, research, and findings data storage for the developement of this profession as a science.

Key words: risk management, patient safety, nursing

\section{METODOLOGÍAS Y HERRAMIENTAS PARA LA SEGURIDAD DEL PACIENTE}

\begin{abstract}
INTRODUCCIÓN

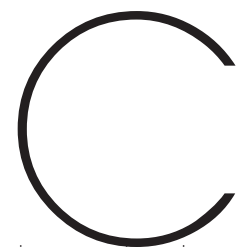

omo parte del cuidado a la salud las personas recibirán en algún momento de su vida atención médica, ya sea en el primer, segundo o tercer nivel de atención; por lo cual, es importante asegurar que dicha atención produzca beneficios y no daños
\end{abstract}

La preocupación por la seguridad del paciente no es un tema nuevo, pero si poco desarrollado. Después del primer estudio sobre eventos adversos llevado a cabo en los años 60's, le siguieron otros estudios que evidenciaron la necesidad de tomar medidas preventivas sobre las fallas en la atención sanitaria. Con base en ello, organizaciones internacionales como la Organización Mundial de la Salud (OMS) y la Organización Panamericana de la Salud (OPS), han propuesto estrategias que ayudan a disminuir o evitar las consecuencias negativas de la atención médica.

Actualmente existen diversas metodologías y herramientas que permiten la gestión de riesgos, facilitan la práctica clínica, y por lo tanto mejoran la calidad de la atención sanitaria. Sin embargo, aún queda mucho por hacer en el área de la seguridad. Con respecto a enfermería, existe una gran área de oportunidad para generar investigación que evidencie cómo y cuándo emplear las diversas metodologías y herramientas, y que proponga nuevas estrategias que generen una asistencia sanitaria más segura.

154 LMN Why Enf Neurol Vol. 15. No. 3 septiembre-diciembre 2016 


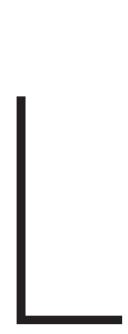

ANTECEDENTES

a seguridad del paciente es un tema de suma importancia para la atención hospitalaria del profesional de enfermería, ya que permite reducir los errores y mejorar la calidad del de los pilares en los que se ha apoyado la práction clínica desde la época hipocrática, sin embargo, se ha enfocado desde una perspectiva ética y no de mejora de la calidad.?

La evidencia científica que se tiene de este tema es escasa, debido a que es un tema relativamente nuevo. Se encuentran registros a partir de la década de los años $60^{2}$ con el primer estudio de eventos adversos realizado por Schimel, en donde describe el tipo y la frecuencia de las complicaciones hospitalarias que se producen en los pacientes; él realizó la investigación en un hospital universitario con una muestra de 1014 pacientes, donde observó que el 20\% sufrió uno o más episodios de complicaciones médicas, a las cuales clasificó de la siguiente manera:

- Reacciones a los procedimientos de diagnóstico.

- Reacciones a fármacos terapéuticos.

- Reacciones a las transfusiones.

- Reacciones a otros procedimientos terapéuticos.

- Infecciones adquiridas.

- Peligros varios de hospital.

En sus resultados reporta que una de las complicaciones que con más frecuencia ocurrió fue la reacción a fármacos terapéuticos. Este estudio fue de gran importancia porque dio pauta a otros países para comenzar a estudiar y documentar sobre los eventos adversos; los cuales más tarde darían lugar a estrategias para mejorar la seguridad del paciente. En el año de 1991 un estudio publicado por Brennan y Colaboradores 4 , indicó que el $4 \%$ de los pacientes hospitalizados sufren algún tipo de daño, y que de estos el 70\% producen una incapacidad temporal y el $14 \%$ son incidentes que terminan en la muerte. Datos clave que no fueron valorados, ya que a pesar de tener estos dos estudios, la seguridad del paciente toma interés por parte del sistema de salud de Estados Unidos a partir de la alta incidencia en eventos adversos ${ }^{5}$ y de la publicación de un nuevo estudio nombrado "errar es de humanos"`, donde describe al error médico como la $8^{a}$ causa de mortalidad en Estados Unidos ${ }^{7.8}$. Y es así que comienzan a realizarse acciones encaminadas a crear una cultura de calidad en la asistencia sanitaria.

En marzo del año 2002 la OMS en su 55a Asamblea Mundial informó tasas muy altas de eventos adversos para diferentes países desarrollados que oscilaron entre 3.2\% y 16.6\%, corroborando el gran problema existente en el ámbito mundial?. Es por ello que en el año 2003 el National Quality Forum (NQF) publicó 30 recomendaciones de prácticas seguras, entre las cuales se encuentra en primer lugar la implementación de la mejora en la cultura de la seguridad del paciente hospitalizado "Años más tarde en 2004, se inicia la campaña "Alianza mundial por la seguridad del paciente" encabezada por la OMS ${ }^{17-13,}$ en la que también se desarrollan iniciativas importantes a nivel internacional, tomando como eje cuatro compromisos para formular una taxonomía que sirva para notificar sucesos adversos y elaborar sistemas de notificación y aprendizaje para facilitar el análisis de las causas que originan errores.

La definición más precisa y actual de la seguridad del paciente es la que brinda la OMS y es considerada como la ausencia de daño real o potencial relacionado con los servicios de salud ${ }^{14}$. Y desde la perspectiva de la atención de salud supone un conjunto de normas, procedimientos, instrumentos y métodos basados en evidencias

Enf Neurol Vol. 15. No. 3 septiembre - diciembre 2016 Wh 155 
científicas destinados a minimizar el riesgo de daño sobre agregado y de eventos adversos en la atención de la salud. Incluye medidas que garantizan prácticas diagnósticas, terapéuticas y cuidados de enfermería seguros, así como de ajuste del medio ambiente, organización y funcionamiento institucional, incluyendo la dotación, competencias y sostenimiento del personal ${ }^{15}$.

A nivel internacional, los sistemas de salud reconocen que la seguridad del paciente es un asunto prioritario y de interés público, tanto por el riesgo a la salud y la vida de las personas como los aspectos éticos y legales. Y los consecuentes hechos para los involucrados, es decir, para el paciente, familiar, médico, enfermera, técnicos, administradores y la sociedad en su conjunto.16

En México en un estudio multicéntrico realizado por Zarate y colaboradores, se evidenció que el evento adverso que se presentó con mayor frecuencia fue el relacionado con el cuidado en un 30.7\%, y que los pacientes más vulnerables a ellos fueron recién nacidos y adultos inconscientes; además el 17.7\% estaban asociados con la medicación y las soluciones parenterales, de estos el 50\% estuvo ligado a la administración (vía, paciente, hora, dosis, entre otros) ${ }^{17}$.

Por lo anterior mejorar la seguridad de los pacientes se ha convertido en un objetivo prioritario en las políticas de calidad de los sistemas sanitarios y se han adoptado estrategias por diversos organismos internacionales para disminuir la ocurrencia de eventos adversos relacionados con la asistencia sanitaria $^{16}$ así como el uso de metodologías y herramientas que garanticen la seguridad del paciente ${ }^{17}$.

Para enfermería Según Zarate ${ }^{18}$ la seguridad es intrínseca al cuidado y a la calidad de éste, ya que implica satisfacer las necesidades y cumplir con las expectativas de las personas y sus familias con la premisa clara de no hacer daño. Hablar de un cuidado seguro implica una práctica profesional basada en evidencia, intencionada, planeada, que proteja y ayude a las personas, creadora de condiciones favorables en el medio ambiente de la persona cuidada.

\section{Metodologías utilizadas en enfermería para la evaluación del evento adverso en la seguridad del paciente}

Actualmente existen diferentes métodos que permiten realizar una evaluación sobre los eventos adversos e incidentes que pueden ocurrir durante la atención del paciente. Algunos de estos métodos tiene una perspectiva diferente, por lo que su enfoque podría dirigirse a la facilidad de uso por el equipo de trabajo, integración a las metodologías de gestión ya implementadas en la organización o a la utilidad en la eliminación del problema de origen que evite la ocurrencia, nuevamente, del evento. ${ }^{19}$

Derivado del interés profundo en mejorar la calidad de atención a nivel general, algunas metodologías que se han utilizado es el análisis de modo de falla, también llamado análisis de causa raíz o análisis de modo falla y efecto (AMFE); el cual consiste en la existencia de múltiples factores que llevan o pueden llevar al error, analiza al mismo desde una perspectiva reactiva centrado en la forma sistémica del error, donde se examina las causas y el entorno que produjeron el incidente o el daño. Este tipo de metodología permite ser dirigida al análisis de identificación, evaluación y prevención de posibles daños futuros. 20,21

Otra metodología empleada para mantener o mejorar la calidad de la atención y la seguridad del paciente es el uso del protocolo de Londres, el cual se encuentra basado en el modelo explicativo de James Rea 22 y que es adaptable

\section{6} 4MN $/ 5 y_{w}$ Enf Neurol Vol. 15. No. 3 septiembre - diciembre 2016 
y útil, para los servicios de salud; ya que permite evaluar los errores en salud de forma organizada, secuencial y lineal para obtener un producto final. Las decisiones que se toman en los niveles directivo y gerencial de la organización se transmiten hacia abajo, a través de los canales departamentales, afectando por ultimo a los sitios de trabajo, favoreciendo así a la creación de condiciones que pueden propiciar conductas seguras de diversa naturaleza.

Para esta metodología se debe seguir una serie de pasos, los cuales permitirán que se presente la naturaleza de la misma y que a continuación se mencionan, según Sánchez 23.

- Identificación y decisión de investigar

- Selección del equipo investigador

- Obtención y organización de información

- Establecimiento de la cronología del incidente

- Identificación de las acciones inseguras

- Recomendaciones y plan de acción

El plan de acción debe incluir la siguiente información

- Priorización de los factores contributivos de acuerdo con su impacto sobre la seguridad futura de los pacientes

- Lista de acciones para enfrentar cada factor contributivo identificado por el equipo investigador

- Responsable de implementar las acciones

- Tiempo de implementación de las acciones

- Recursos necesarios

- Seguimiento a la ejecución del plan

- Cierre formal cuando la implementación se haya efectuado

- Fecha de seguimiento para evaluar la efectividad del plan de acción

Otro método existente es el llamado ANCLA, utilizado para la investigación del análisis de la evaluación del evento adverso. Este contempla cinco niveles de investigación que permiten identificar los actos inseguros, las precondiciones para actos inseguros (basado en el Modelo SHELL), la supervisión, las influencias organizacionales y la influencia de la legislación y el estado: como herramienta para definir las recomendaciones adecuadas y su respectivo plan de acción. ${ }^{24}$ En su implementación es conveniente considerar dos fases; una de aproximación inicial, en la cual se clasifica el evento y se obtiene información y una segunda, en la cual se investiga el evento (aplicación del método), se documenta y se hace un reporte final.

Cabe mencionar que después de la búsqueda en las diferentes bases de datos se encontró poca evidencia que relacionara el uso de estas metodologías dentro del ámbito de investigación por parte de la disciplina de enfermería, lo que se convierte en un área de oportunidad para los futuros investigadores.

\section{Herramientas para la seguridad del paciente}

En la gestión de la seguridad del paciente durante la atención sanitaria u hospitalaria, se cuenta con herramientas que facilitan la práctica clínica; alguna de ellas son las guías sobre seguridad del paciente que abordan varias líneas de actuación, con las principales recomendaciones que los profesionales en enfermería deben seguir en cada una de ellas, para incrementar la efectividad de los procesos y garantizar la seguridad de los pacientes durante la asistencia; principalmente en situaciones de urgencias y emergencias en el que el entorno cambiante y el factor tiempo incorporan mayores riesgos a la atención clínica.

Por otra parte, las guías de práctica clínica (GPC) son un conjunto de recomendaciones desarrolladas de manera sistemática, con el objetivo de guiar a los profesionales y a los enfermos en el proceso de toma de decisiones y elegir qué intervenciones sanitarias son más adecuadas en el abordaje de una condición clínica específica en circunstancias sanitarias concretas.

Enf Neurol Vol. 15. No. 3 septiembre - diciembre 2016 
Las GPC ayudan a los profesionales a asimilar, evaluar e implantar y a su vez recopilar mayor evidencia científica disponible y las opiniones basadas en la mejor práctica clínica. El propósito de hacer unas recomendaciones explícitas es influir en la práctica, por lo que éstas han de tener validez tanto interna como externa y ser aplicables. En la actualidad no existe ninguna GPC que se relacione exclusivamente con la seguridad del paciente, independientemente del proceso patológico que padezca. 25,26 Lo anterior puede estar relacionado con que la ocurrencia de fallas que conducen a la incidencia de eventos adversos incluye toda una serie de situaciones tanto en la estructura de la organización como en los procesos.

Otra de las herramientas muy utilizadas en la actualidad son los estándares de calidad, esto ha adquirido gran relevancia en los últimos años tanto para los pacientes y sus familias quienes desean sentirse seguros y confiados con los cuidados sanitarios recibidos, como para los gestores y profesionales que desean ofrecer una asistencia sanitaria efectiva y eficiente. Basta revisar la literatura científica para comprobar la importancia que ha cobrado en los últimos años los temas relacionados con la seguridad de los pacientes. La propia OMS establece la Alianza Mundial para el abordaje de la Seguridad del Paciente en cuanto a los sistemas sanitarios. ${ }^{27}$

La gestión de la seguridad es parte de la calidad. Y dentro de la calidad hay estándares e indicadores que ayudan a la mejora continua de la seguridad del paciente, así también normas sanitarias que se encuentran incluidas en la ley sanitaria de cada país, éstas son herramientas que ya están establecidas y el no cumplimiento de ellas, trae consigo consecuencias legales. No debe olvidarse que una de las mejores herramientas es el conocimiento que cada profesional debe tener, adquirido durante su formación teórica y reforzado durante su práctica, para estar en las condiciones de proporcionar un excelente cuidado y que la seguridad del paciente nunca se vea comprometida.

\section{CONCLUSIONES}

- rabajar en la calidad y seguridad de la atención sanitaria, no sólo reduce los costos, también salva vidas. Por lo cual hacer uso de metodologías como el análisis de causa raíz, el protocolo de Londres, y el método ANCLA, así como herramientas establecidas por organismos internacionales, utilizadas en diferentes escenarios del ámbito sanitario, como "los estándares de calidad de los procesos, las guías de práctica clínica, y el índice de seguridad hospitalaria" permitirán proporcionar atención segura y de calidad.

Al revisar la literatura en torno al tema de seguridad del paciente y las intervenciones de enfermería, se hace notar la ausencia de documentos científicos que evidencien lo que las enfermeras hacen todos los días en su práctica profesional para disminuir o controlar los riesgos en la atención sanitaria. Porque si bien es cierto la gestión de los riesgos es un trabajo multidisciplinario, la enfermera juega un papel muy importante, al estar presente en todos los niveles de los sistemas de salud, como gestora, líder, administradora, cuidadora, orientadora, entre otros. El documentar los resultados del uso de dichas metodologías y herramientas permitirá que otros profesionales adopten las que mejor se adapten a las necesidades de los pacientes, que se creen políticas públicas en torno al tema de la seguridad, que se fortalezca la cultura de la prevención, que el personal sanitario pueda actuar de manera proactiva y fundamente su práctica en la mejor evidencia científica; contribuyendo de esta manera al fortalecimiento de la práctica de la enfermería y al desarrollo de ésta como ciencia del cuidado.

\section{8} MMN Why Enf Neurol Vol. 15. No. 3 septiembre - diciembre 2016 


\section{REFERENCIAS BIBLIOGRÁFICAS}

1. Aranaz J, Limón R, Aibar C, Miralles J, Vitaller J, Terol E, et al. Luces y sombras en la seguridad del paciente: estudio y desarrollo de estratégias. Gac Sanit. 2008: 22(1): 198-204

2. Muiño $A$, Jiménez $A$, Pinilla $B$, Durán $M$, Cabrera F, Rodriguez M. An Med. Interna. Madrid. 2007: 24(12):602-606.

3. Schimel M. The hazards of hospitalization. Qual. Saf. Health Care. 2003: $12 ; 58-63$

4. Brennan T, Leape L, Laird M, Hebert L, Localio A, Lawthers A, et al. Incidence of adverse events and negligence in hospitalized patients: results of the Harvard Medical Practice Study I. N Engl J Med 1997; 324:370-6.

5. The Task Force: Quality interagency coordination (QulC) Task Force. US Government; 1999

6. Kohn L, Corrigan J, Donaldson MS (Eds.). To err is human: Building a safer health system. Washington, DC: National Academy Press; 1999.

7. Astolfo F. La seguridad clínica de los pacientes: entendiendo el problema. Revista Colombia Médica. 2005: 36(2): 130-133.

8. Martín D, Cabré P. Aspectos éticos y legales sobre la seguridad del paciente. Rev Bio y der. 2015; número extra: 146-159.

9. 55a Asamblea Mundial de la Salud. Tercer informe de la Comisión A (Serie A55/52), Ginebra, OMS, 2002. (Acceso: 02-06-2016.) Disponible en: http:// apps.who.int/gb/archive/pdf_files/EB110/seb 1102.pdf.

10. The National Quallity Forum. Safe practices for better healthcare: a consensus report. Washington DC: The National Quality Forum; 2003

11. Nora L, Studwell S. Improving patient safety. What can detailed case analysis tell us? Neurology. 2005; 65: 1154-5.

12. Sage W. Malpractice liability, patient safety and the personification of medical injury; opportunities for academic medicine. Academic Medicine. 2006; 81: 823-6.

13. Campos M, Carrillo A. Reporte de Errores Médicos como estrategia para la Prevención de Eventos Adversos. Revista CONAMED. 2008; 13:17-22

14. World Health Organization. World alliance for patient safety: forward program Geneva: WHO Press; 2004

15. Castañeda H, Garza H, González S, Pineda Z, Acevedo P. Aguilera P, Percepción de la cultura de la seguridad de los pacientes por personal de enfermería. Ciencia y Enfermería. 2013; XIX(2): 77-88

16. Muiño M, Jiménez M, Pinilla L, Durán G, Cabrera A, Rodríguez PM. An Med. Interna. Madrid. 2007; 24(12):602-606.

17. Zárate G.R. La seguridad del paciente un compromiso para la enfermería univeritaria. Revista Enfermería Universitaria ENEO-UNAM. 2007; 4(3):3-6.
18. Zárate-Grajales R, Olvera-Arreola S, Hernández-Cantoral A, Hernández C.S, Sánchez-Ángeles S, Valdez L.R, et al. Factores relacionados con eventos adversos reportados por enfermería en unidades de cuidados intensivos Enfermería Universitaria. 2015; 12(2):63-72

18. Minsalud. Evaluación de la frecuencia de eventos adversos y monitoreo de aspectos claves relacionados con la seguridad del paciente. Paquetes instruccionales; guía técnica "buenas prácticas para la seguridad de paciente en la atención en salud." (acceso: 04/06/2016). Disponible: https:// uww.minsalud.gov.co/sites/rid/Lists/BibliotecaDigital/RIDE/DE/CA/Eva/uar-fre cuencia-ventos-adversos.palf.

19. Consuegra M. O. Metodología AMFE como herramienta de gestión de riesgo en un hospital universitario. Cuadernos Latinoamericanos de Administración. 20(11): 37-50(acceso 03/06/16). Disponible: http://wnw uelbosque.edu.co/sites/default/files/publicaciones/revistas/cuadernos latinoamericanos_administracion/volumenXI_numero20_2015/05_Metodologia_Vol-X-Num-20.pdf

20. Dirección general de calidad y educación en salud. Guía Técnica para el Análisis Causa - Raíz de Eventos Adversos en hospitales 2013. Subsecretaria de integración y desarrollo del sector salud. (acceso 03/06/16). Disponible en: hitp:// www.calidad.salud.gob.mx/site/calidad/docs/dsp-sp_00A.pdt

21. Trujillo C. Manual de seguridad del usuario. Hospital Nazareth Nivel. Bogotá 2013. Disponible en: http://mww.esenazareth.gov.co/hnintranet/Calidad/ RGSC014\%20Manual\%20de\%20Seguridad\%20de/\%20Usuario\%20-\%20v2.pdf

22. Sánchez J. Gestión de notificaciones de errores de medicación por entidades regulatorias. Rev. colomb. enferm. 10 (10); ABRIL 2015(ACCESO 03/06/2016) 16-26. Disponible: hittp://mww.uelbosque.edu.co/sites/default/ files/publicaciones/revistas/revista_colombiana_enfermeria/volumen10/003 articulo-1-Vo/10\%20A10.pdt

23. ESE Hospital San Rafael. Programa de seguridad del paciente Disponible: $\quad h i t p: / / m u w$.esehospitalsanrafael-pueb/orico-risaralda.gov.co/ apc-aa- HYPERLINK "hittp:// mww.esehospitalsanrafael-pueb/orico-risaralda gov.co/apc-aa-files/62363965306463366236623435616365/programa-de-seguridad-del-paciente.pdf'files/62363965306463366236623435616365/programa-de-seguridad-del-paciente.pdf

24. Huntingdon Valley, PA, Institute for safe medication practices. ISMP's list of confused drug names.

25. Corral S, Guerrero A. Utilización de CMBD como herramienta para la detección de acontecimientos adversos inducidos por medicamentos Farm Hosp ,2004 28(4): 258-265

26. Estándares de calidad de cuidados para la seguridad del paciente en los hospitales del SNS. proyecto SENEca: informe técnico 2008. Madrid Ministerio de Sanidad y política Social; 2009 